\title{
Optical power transfer and communication methods for wireless implantable sensing platforms
}

Muhammad Mujeeb-U-Rahman

Dvin Adalian

Chieh-Feng Chang

Axel Scherer

\section{SPIE.}




\title{
Optical power transfer and communication methods for wireless implantable sensing platforms
}

\author{
Muhammad Mujeeb-U-Rahman, ${ }^{*}$ Dvin Adalian, Chieh-Feng Chang, and Axel Scherer \\ California Institute of Technology, Electrical Engineering, 1200 East California Boulevard, Pasadena, California 91125, United States
}

\begin{abstract}
Ultrasmall scale implants have recently attracted focus as valuable tools for monitoring both acute and chronic diseases. Semiconductor optical technologies are the key to miniaturizing these devices to the longsought sub-mm scale, which will enable long-term use of these devices for medical applications. This can also enable the use of multiple implantable devices concurrently to form a true body area network of sensors. We demonstrate optical power transfer techniques and methods to effectively harness this power for implantable devices. Furthermore, we also present methods for optical data transfer from such implants. Simultaneous use of these technologies can result in miniaturized sensing platforms that can allow for large-scale use of such systems in real world applications. @ 2015 Society of Photo-Optical Instrumentation Engineers (SPIE) [DOI: 10.1117/1.JBO.20.9.095012]
\end{abstract}

Keywords: implant; sensors; wireless; optical; photovoltaics.

Paper 150236PRR received Apr. 8, 2015; accepted for publication Aug. 25, 2015; published online Sep. 25, 2015.

\section{Introduction}

The application of microelectronic technologies to medicine has the potential to revolutionize the field of biomedical engineering. ${ }^{1}$ The advent of smart and ultrasmall (sub-mm) wireless sensing implants can bring an entirely new type of healthcare paradigm to bear as well as improve biomedical research and diagnostics. ${ }^{2}$ This size scale will make the implantation and subsequent removal of such devices easier and cheaper. It will also minimize implantation tissue damage and hence decrease the foreign body immune reaction. ${ }^{3}$ The miniaturization of these functional implants will also permit these devices to achieve their long-term potential for continuous health monitoring.

A fully functional implant consists of a sensor, control circuit, power component, and communication component. ${ }^{4}$ Every component of an implant needs to be optimized for volume, and this work will demonstrate a significant advancement in that effort. There are several examples in literature which demonstrate miniaturization of some components such as sensors ${ }^{5}$ and control functions. ${ }^{6}$ Another important step to permit miniaturization is utilizing shorter wavelength wireless techniques for power transfer and communication. ${ }^{4}$ Electrical methods (i.e., inductive coupling and radio frequency links) have been applied before to power and communicate with such devices, including neural implants, ${ }^{7}$ retinal implants, ${ }^{8}$ and metabolic sensors. ${ }^{9}$ The signal frequencies for such systems are limited to the near $\mathrm{GHz}$ range by regulatory requirements and in vivo loss. ${ }^{10}$ Hence, the fundamental nature of wavelength dependence for electrical antenna design forces a limit on miniaturization of those systems. Optical devices for wireless powering and communication, on the other hand, can be easily miniaturized to sub-mm scale without seriously compromising their efficiencies. ${ }^{11}$ Hence, optical methods are very attractive to use for designing implants when extreme miniaturization is desired. These methods can be used for both power transfer ${ }^{12}$ and telemetry. ${ }^{13}$

\footnotetext{
*Address all correspondence to: Muhammad Mujeeb-U-Rahman, E-mail:
} mrahman@caltech.edu
Using integrated systems to achieve wireless power transfer and communication functions is a key step to decrease the footprint for the wireless implants. In this work, we demonstrate a complimentary metal oxide semiconductor (CMOS) integrated power harvesting system for wirelessly powering a miniaturized implant chip. Furthermore, we provide an example of optical data communication using an ultrasmall vertical cavity surface emitting laser (VCSEL) on the same platform, thus demonstrating the possibility of using optical methods for both power transfer and communication. We start this approach by considering the integrated system design, choices of materials, and operating wavelengths. Then we will consider the best optoelectronic design practices to optimize the photovoltaic (PV) response and apply these considerations into system design parameters for a manufactured CMOS-based design. Finally, we will provide our measurement results for a completed CMOS design for power transfer and a microlaser-based system for telemetry. The ultimate goal of this work is to advance the realization of extremely miniaturized and economical sensor systems which are in high demand for a wide variety of applications.

\section{System Design}

The wireless device in this work is designed for implantation under the skin in the subcutaneous region. The device has to be miniaturized to near-mm size scale to minimize the foreign body response. ${ }^{3}$ The goal is to use an integrated system for most functions and minimize the use of discrete components. This allows for extreme size reduction while maintaining a significantly low cost. The implantable device is comprised of control and processing electronics (Potentiostat), along with electrochemical sensing units, optical powering, and optical communication systems on the same platform. ${ }^{14}$ An external reader unit is used to power the implant via an external laser and also has a photodetector to collect the telemetry signal from the implant. The complete setup is shown in Fig. 1.

$1083-3668 / 2015 / \$ 25.00$ (C) 2015 SPIE 


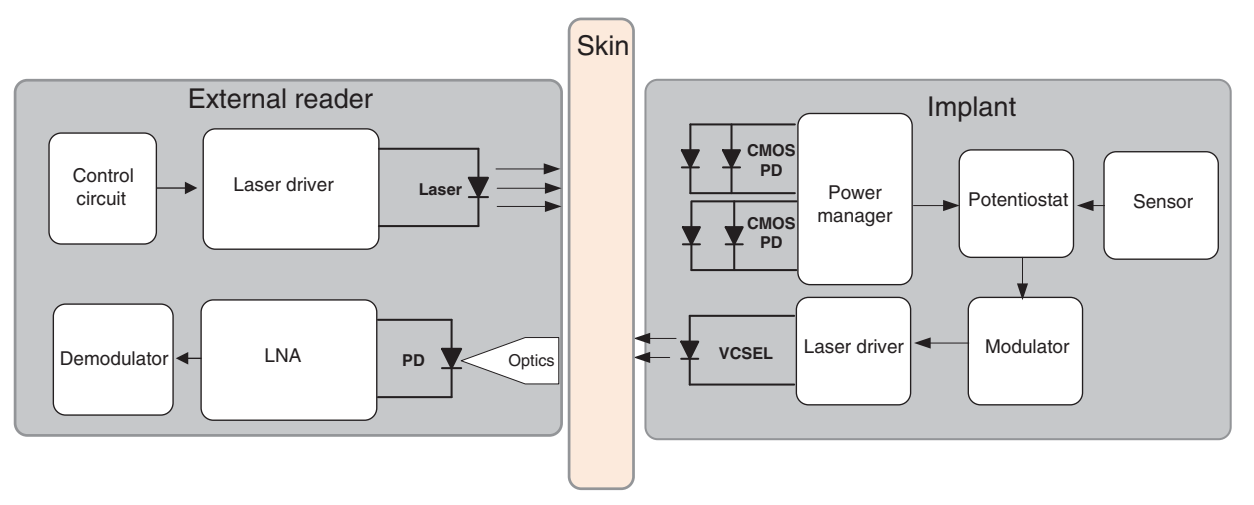

Fig. 1 Complete system depicting optical power harvesting and optical communication system.

Silicon-based integrated electronics (CMOS) is the industry standard for ultrasmall integrated systems. ${ }^{15}$ CMOS technology provides on-chip control and processing circuitry with an extremely small foot print, high reliability, and low cost. ${ }^{16}$ CMOS-based PV power harvesting systems can be much smaller in size compared to other power harvesting methods. ${ }^{17}$ Silicon is not an ideal PV material with its indirect bandgap of $1.1 \mathrm{eV}$ which is best suited to absorb photons of $1.1 \mu \mathrm{m}$ and smaller wavelengths, but it is by far the cheapest semiconductor material. ${ }^{11}$ The photogenerated current inside any material depends upon the intensity of light at that particular depth as given in Eq. (1). ${ }^{11}$

$I(x)=I_{\mathrm{o}} \mathrm{e}^{-\alpha(\lambda) x}$.

In this equation, $\alpha$ is the absorption depth of light of wavelength $\lambda, x$ is the actual depth, and $I$ is the photogenerated current given that $I_{\mathrm{o}}$ is the photogenerated current at the surface $(x=0)$. The absorption depth in silicon ranges from a few microns to tens of microns into the infrared regime. ${ }^{18}$

For optimum performance of implantable systems, the wavelength of the optical signal has to be chosen to minimize absorption and scattering in the biological media (e.g., skin and tissue) while maximizing absorption in the power harvesting subsystem without creating detrimental heating. To this end, the optical properties of in vivo tissue have to be understood to make the choice of wavelength.

Human skin tissue has an intricate structure consisting of three major layers: the epidermis, dermis, and hypodermis, as shown in Fig. $2 .{ }^{19}$ The top layer is the epidermis $(\sim 100 \mu \mathrm{m})$ and its top subdivision is the stratum corneum which has a refractive index of 1.55 and is commonly tens of microns thick. ${ }^{20}$ The dermis thickness varies widely around 2 to $4 \mathrm{~mm}$ and has an order of magnitude less absorption and scattering than the epidermis. The subcutis (or hypodermis) is below the dermis and also has variable thickness in the mm range and is the prime location for implants which are intended to measure the composition of interstitial fluid. $^{21}$

The absorption of light in skin has a significant draining effect on PV powering of implants. In the near IR spectrum, the material differences between the different types of skin have less consequence than the total amount of water or hemoglobin present, which are the dominant absorbers. ${ }^{22}$ In general, muscle tissues are simpler and are modeled using a similar scheme with a lower number of layers than those needed for skin. ${ }^{23}$ near infrared (NIR) light absorption in human skin is shown in Fig. $3 .^{24}$

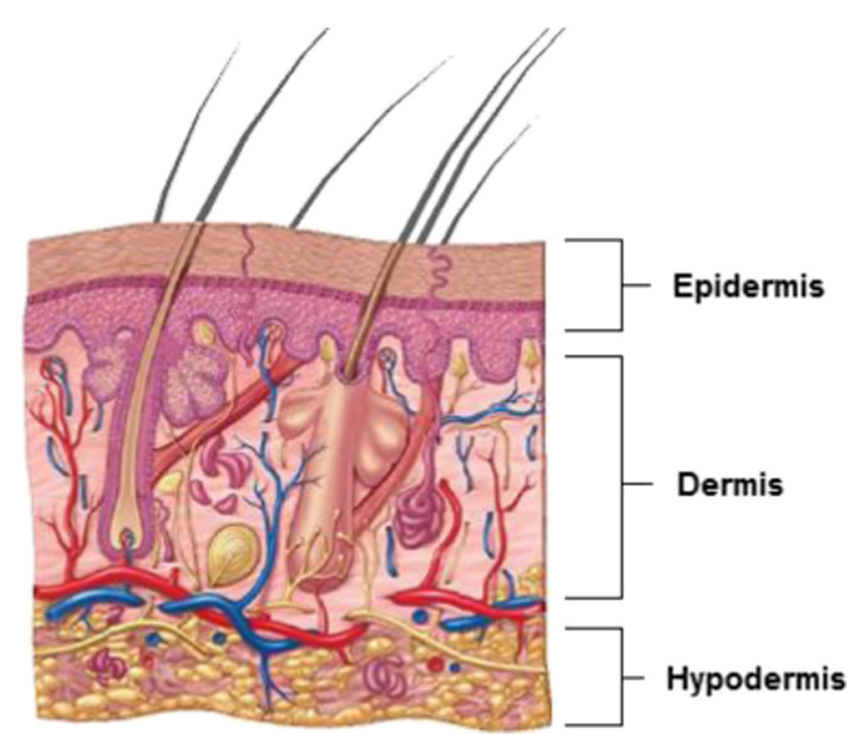

Fig. 2 Anatomy of human skin. ${ }^{19}$

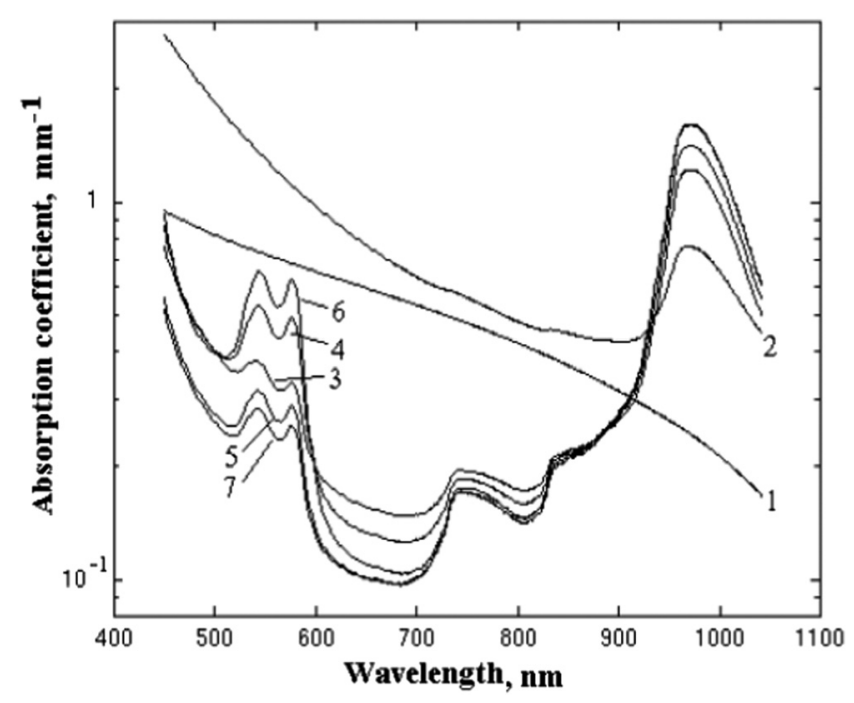

Fig. 3 Wavelength-dependent absorption of different components of human skin, ${ }^{24}$ (1-stratum corneum, 2-living epidermis, 3-papillary dermis, 4-upper blood net dermis, 5-reticular dermis, 6-deep blood net dermis, 7-subcutaneous fat). 


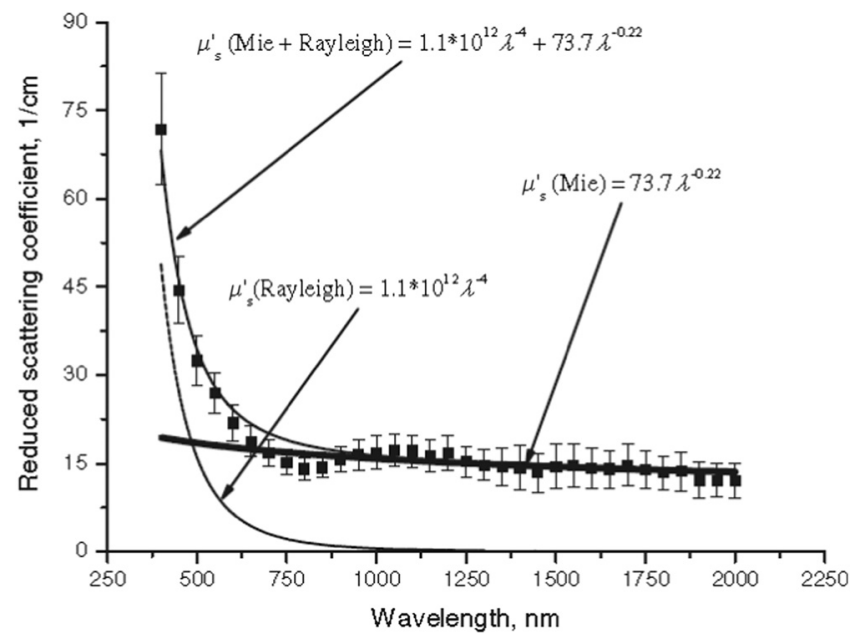

Fig. 4 Scattering coefficients of different layers of the skin tissue and fitting results from Mie and Rayleigh theories which describe the spectral dependence of the reduced scattering coefficient of human skin in vitro and its approximation by power law. ${ }^{25}$

Scattering is also a significant factor in optical system design and results from many different types of particles in the tissue. Scattering is also wavelength dependent since there are many different sizes of particles present. Scattering in tissue layers near the skin surface in the NIR regime is shown in Fig. $4{ }^{25}$ It is evident that scattering increases with a decrease in wavelength, but is fairly constant in the range of 750 to $1000 \mathrm{~nm}$.

The scattering coefficient $(\mu s)$ is a parameter defined to mathematically include scattering as a loss mechanism as in the light transport equation, ${ }^{26}$

$I(x)=I_{o} \mathrm{e}^{-[\alpha(\lambda)+\mu s] x}$.

The reduced scattering coefficient is defined to include the effect of anisotropy of the scattering properties of tissue. Another parameter, $g$, is added to the model which is called the coefficient of anisotropy and varies between 0.69 and 0.99 for biological tissues $\left(g=0\right.$ for isotropic media). ${ }^{27}$ Finally, a reduced coefficient of scattering is defined $\left(\mu_{s}^{\prime}\right)$, as in the following equation: ${ }^{27}$

$\mu_{s}^{\prime}=(1-g) \mu_{s}$

Reflectance is another significant loss mechanism. The magnitude of reflectance depends upon skin type and should be experimentally determined. ${ }^{28}$ Due to the multilayer structure, reflections must be accounted for at multiple interfaces in the skin. However, the net effect of reflection can be captured using a properly defined reflection coefficient under suitable conditions. ${ }^{29}$ The above analysis suggests that the NIR region (also known as the "therapeutic window") in the optical spectrum has minimal overall loss and can provide maximal signal transfer to a subcutaneous implant. ${ }^{27}$ The overall transmission equation for light in this wavelength region can be written as

$I(x)=\Gamma I_{o} \mathrm{e}^{\left.-\left[\alpha(\lambda)+\mu_{s}^{\prime}\right)\right] x}$.

Here, $\Gamma$ is the fraction of light that enters skin from the total light intensity that shines on its surface (incorporating reflection). Using typical parameters of $3 \mathrm{~mm}$ for thick skin, for $850 \mathrm{~nm}$ wavelength i.e., $\alpha=0.1 \mathrm{~mm}^{-1}, \quad \mu_{s}^{\prime}=1.6 \mathrm{~mm}^{-1}$,
$\Gamma=0.7,{ }^{22}$ we can calculate that the efficiency of light reaching the CMOS implant will be quite low, $0.27 \%$. Therefore, the majority of the light gets absorbed, reflected, or scattered before reaching the CMOS device, thus high-light intensity is needed to generate appreciable power in the device.

The above-mentioned therapeutic wavelength range can also be used for data telemetry. But silicon is not an efficient active element for manipulating optical frequency signals. ${ }^{11}$ Hence, we demonstrate communication using a compound semiconductorbased VCSEL from ULM Photonics. ${ }^{30}$ These devices have very low-threshold current and one of the lowest threshold voltages for devices in our desired wavelength range in the therapeutic window. An external photodetector from Thorlabs ${ }^{31}$ was used to detect the VCSEL signal.

\subsection{Optoelectronic Analysis}

Optoelectronic analysis is required to optimize the properties of CMOS-based electronic devices as PV systems. The analysis begins with calculating the open circuit voltage of a $\mathrm{p}-\mathrm{n}$ junction PV device which is given by following relationship: ${ }^{11}$

$V_{\mathrm{OC}}=\frac{n k T}{q} \ln \left[\frac{J_{\mathrm{sc}}}{J_{O}}+1\right]$

Here, $J_{\mathrm{sc}}$ is the short circuit current which is related to the photogenerated current and $J_{O}$ is the reverse saturation current of the $\mathrm{p}-\mathrm{n}$ junction. The logarithmic dependence of voltage to the ratio of these currents shows that high-light intensities are required to create a large number of photogenerated carriers (photocurrent) while maintaining a very low-reverse saturation current. Thus, critical design aspects of the absorber are the fabrication quality of the junction (n) as well as the device layout design which determines the level of reverse saturation current.

The photogenerated current from a $\mathrm{p}-\mathrm{n}$ junction PV device comes from electron-hole pair generation due to photon absorption/excitation. For most cells with small series resistance, the short circuit current and photogenerated current are almost equal and the short circuit current $\left(J_{\mathrm{sc}}\right)$ can be determined from the photogenerated current $\left(J_{\mathrm{ph}}\right)$, as shown by the following relationship: ${ }^{11}$

$J_{\mathrm{sc}}=q\left(L_{n}+L_{p}+W_{d}\right) G=q \mathrm{NQE}(\lambda)=J_{\mathrm{ph}}(\lambda)$

Here, QE is external quantum efficiency, $N$ is number of photons, and $q$ is the electronic charge. Clearly, maximizing the available region where photons can be absorbed and resulting electron-hole pairs can be collected before recombination is paramount. This area depends upon the depletion region width of a p-n junction device $\left(W_{d}\right)$ as well as the diffusion length of minority carriers in the $p\left(L_{p}\right)$ and $n\left(L_{n}\right)$ regions. The area of the PV cell is denoted $A$, and $G$ is the rate of photogenerated electron-hole pairs. Since the rate of photogenerated electronhole pairs for a given wavelength depends linearly upon the light intensity, we utilized a laser for its ability to deliver a narrow intensity enabling both high current and high-cell potential.

For a PV device under load which has a maximum capacity to generate photocurrent $J_{\mathrm{sc}}$, only a portion of it can be provided to the load for a nonzero voltage $V_{\text {loaded }}$ across the device. This is given by the following equation: ${ }^{11}$ 
$V_{\text {loaded }}=\frac{n k T}{q} \ln \left[\frac{J_{\mathrm{SC}}-J_{\text {load }}}{J_{O}}+1\right]$.

In theory, the maximum available power is given by a product of the maximum output voltage (open circuit voltage $V_{\text {oc }}$ ) and the maximum available current (short circuit current $J_{\mathrm{sc}}$ ). However, in practice, operating at this maximum is not possible due to the loading effect from the device's impedance. To correct for this, a fill factor $(\mathrm{FF})$ is defined to include the effect of loading on the cell. It is described by ${ }^{11}$

$\mathrm{FF}=\frac{J_{m} V_{m}}{J_{\mathrm{sc}} V_{\mathrm{oc}}}$

Here, $J_{m}$ and $V_{m}$ are the output current density and output voltage at the maximum power point. The FF is often used to define the maximum cell output in terms of the cell characteristics as ${ }^{11}$

$P_{m}=\mathrm{FF} V_{\mathrm{oc}} I_{\mathrm{sc}}$.

The maximum power point is reached when the cell is loaded with its characteristic load impedance ${ }^{11}$

$R_{\mathrm{ch}}=\frac{V_{m}}{I_{m}}$

Thus, maximizing FF is as important as maximizing $V_{\mathrm{oc}}$ and $I_{\text {sc }}$ to get optimal cell output power. FF is maximized by decreasing the amount of photogenerated current not converting into cell output which is lowering the available voltage and current. To do this, we must optimize the system geometry as will be discussed in the next section.

\subsection{System Implementation}

For system implementation, the CMOS designs are physically realized using TSMC $250 \mathrm{~nm}$ standard CMOS technology offered through MOSIS service. Different available junctions in this CMOS process are shown in Figs. 5 and 6.

Generally, $n-p$ diodes are expected to have a better spectral response compared with $\mathrm{p}-\mathrm{n}$ diodes due to higher electron mobility and lower hole mobility. ${ }^{11}$ Also, lightly doped regions have low-recombination rates (because of larger minority carrier diffusion lengths) and thus perform better than highly doped regions. Additionally, each type of junction is expected to have a slightly different quantum efficiency and hence different responsivity (amps/watt) to light input. From a comparison between different photodiodes in the literature, ${ }^{32}$ it is evident

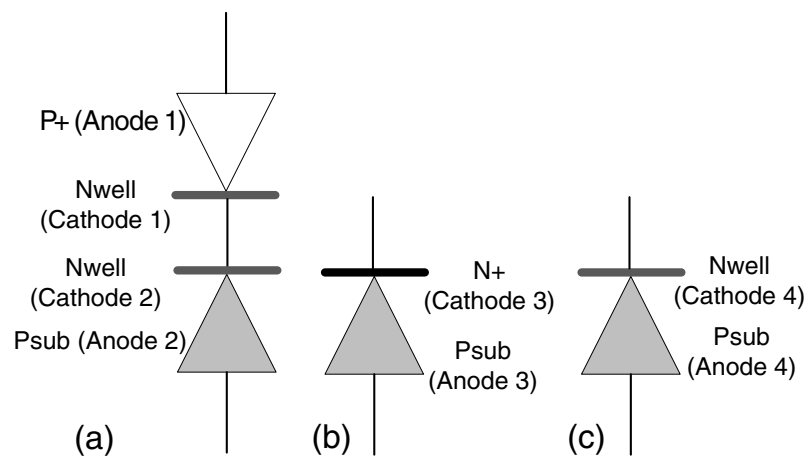

Fig. 6 Types of junction diodes in standard CMOS: (a) P+/NWell, (b) N+/Psub, and (c) Nwell/Psub.

that the n-well/p structure is the best option for maximizing current. Some standard CMOS processes have only basic n- and p-wells, whereas others have both standard and deep n- and p-wells which are crucial to consider since deep wells are very useful for implementing high voltages in standard CMOS.

An obstacle to achieve high voltages in CMOS is the common shared substrate that causes leakage when stacking PV devices. ${ }^{33}$ For series stacking, none of the possible $n+/ p$ substrate, $\mathrm{n}$-well/p substrate, and $\mathrm{p}+/ \mathrm{n}$-well diode designs can be used due to the parasitic buried diode junctions as shown in Fig. 7. It is important to note that CMOS substrates are normally p-doped and hence have to be connected to the lowest potential in the circuit for proper operation of the transistors.

Different solutions have been previously proposed, including the use of external components to compensate for substrate voltage leakage ${ }^{33}$ or the use of DC-DC converters to boost the available voltage. ${ }^{34}$ However, most of these correction methods require a large area or complex circuit design which increases system complexity and hence size requirements. In our design, we utilized the deep-well type structures since these are successfully used to electrically isolate different parts of circuits in RF applications. ${ }^{15}$ For a standard CMOS process with deep NWell regions, different types of doped regions (lightly doped wells, highly doped contact regions, very lightly doped substrates, and so on) are available to build a PV cell. These are shown in Figs. 8 and 9 [ohmic contacts to $\mathrm{P}$ and $\mathrm{N}$ regions are made by respective highly doped regions $(\mathrm{P}+$ and $\mathrm{N}+)$ ].

The result of the parasitic bipolar junction transistor structures is leakage of current to the substrate and a decrease of the percentage of photocurrent available for the load. ${ }^{33}$ Substrate leakage also limits the current of the stacked devices even when large photocollectors are used. In this design, three diodes

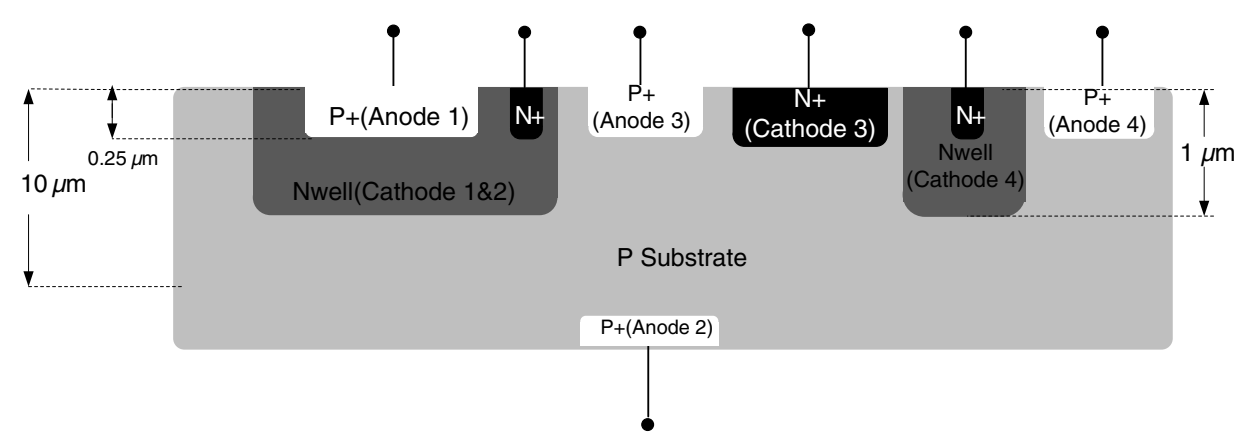

Fig. 5 Types of junctions in standard complementary metal oxide semiconductor (CMOS) devices. 

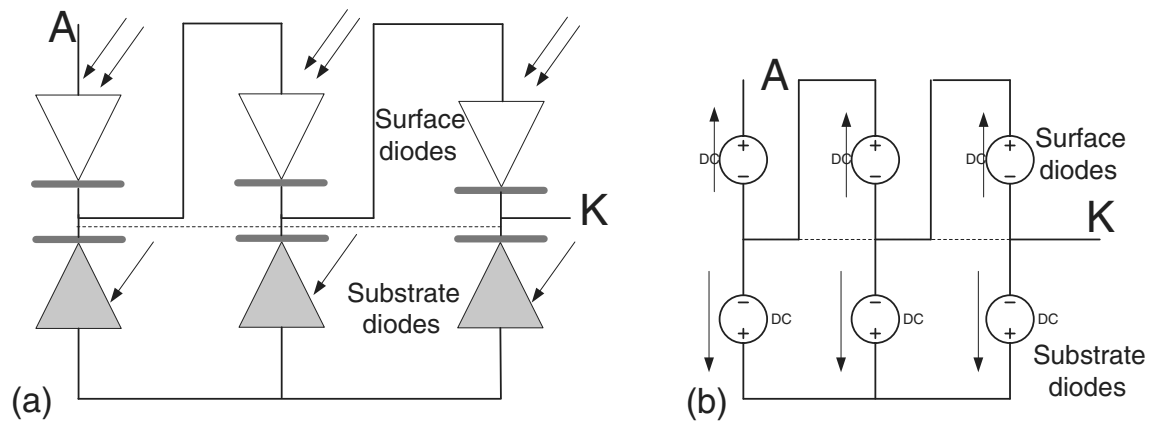

Fig. 7 Effect of parasitic devices on diode stacking: (a) diagram of diodes showing parasitic junction shorting (dotted line) and (b) equivalent circuit showing resulting voltage sources shorting (dotted line).

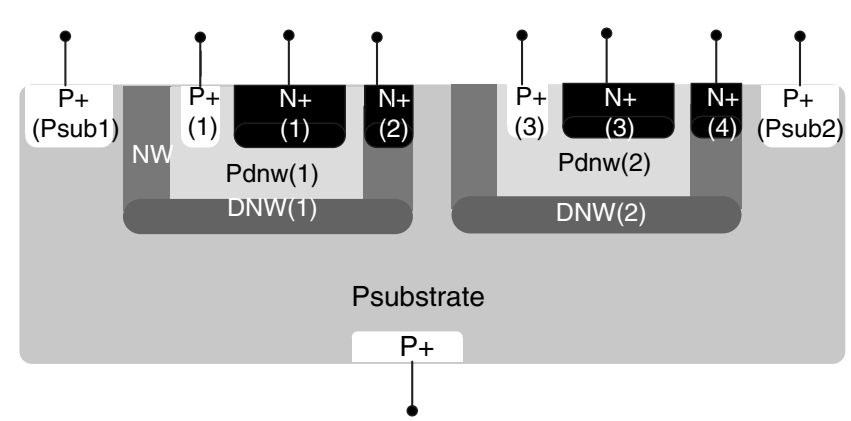

Fig. 8 Types of junctions in deep NWell CMOS process (DNW is used to abbreviate "deep NWell," NW is for "NWell," Pdnw is for "P region in deep NWell").

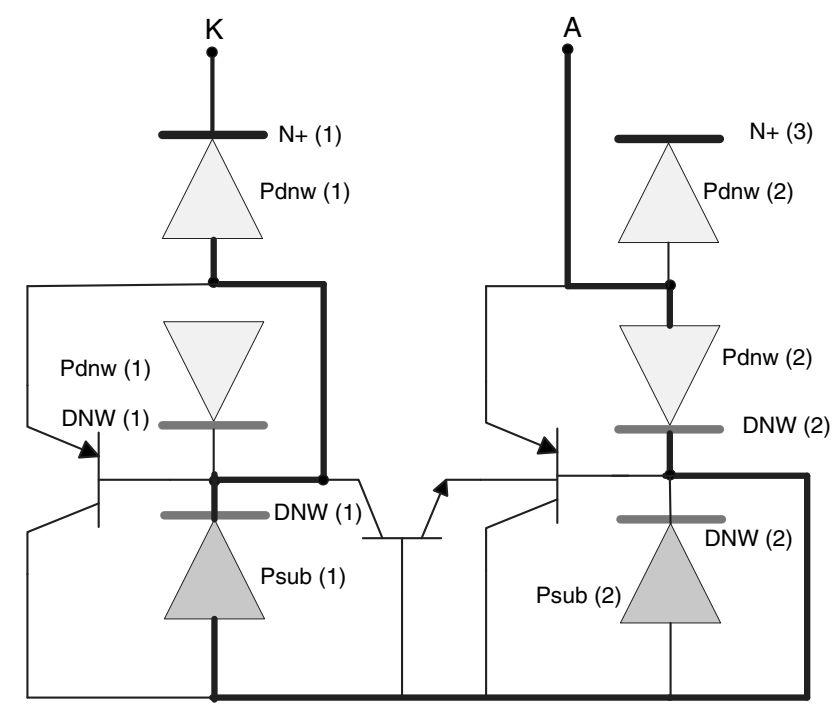

Fig. 9 Devices in deep NWell-based CMOS process (junction diodes and parasitic BJTs).

can be stacked in series by using the topology in Fig. 9 (shown by the darker connections). This method of stacking is challenging due to the current matching requirements between different types of diodes. However, recursive design testing enabled us to achieve sufficient matching between the devices and deliver current to power the electronic control system. Thin-metal contacts (lower level metals in the CMOS process) are used for current collection to maximize the collection of photogenerated carriers. A PV device on the actual CMOS die is shown in Fig. 10.

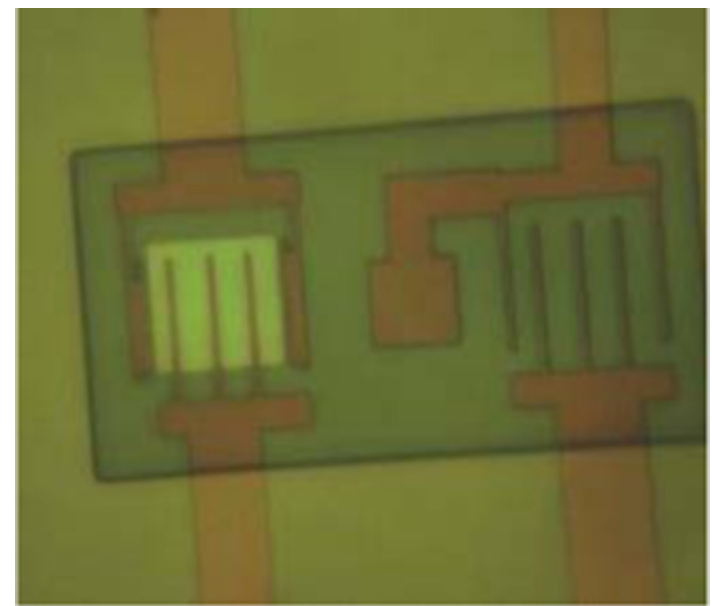

Fig. 10 Micrograph of CMOS photovoltaics (PVs) utilizing deep NWell-based design.

As mentioned earlier, optical telemetry links are an attractive option for ultrasmall footprint requirements. ${ }^{35} \mathrm{~A}$ VCSEL is the smallest commercially available laser and hence is a clear choice for optical communication at this size scale. ${ }^{36}$ The laser can be further miniaturized by thinning down the compound semiconductor handle substrate made of GaAs. In addition to mechanical polishing, the substrate can be etched away with a piranha solution of $4 \mathrm{H}_{2} \mathrm{SO}_{4}: 1 \mathrm{H}_{2} \mathrm{O}_{2}: 1 \mathrm{H}_{2} \mathrm{O}_{2}$, while the front side is protected with wax or a similarly inert layer. In our experiment, the etch rate in such a chemistry was determined to be $\sim 1 \mu \mathrm{m} / \mathrm{min}$. This thinning process facilitates subsequent bonding and fabrication steps since the resulting device surface has more surface roughness. A thinned down VCSEL along with its mirror structure revealed through focused ion beam etching and HF etching is shown in Fig. 11.

\section{Testing and Results}

The setup used for PV device testing consists of a high-power $805 \mathrm{~nm}$ IR laser $(0.8 \mathrm{~W})$, a Keithley 2400 source meter and a balanced optical table setup for alignment. An Agilent/HP 4155 semiconductor parameter analyzer in an electrical probe station was used to do electrical testing of the devices.

A similar testing scheme was used for measuring the VCSEL. Insulating glue was used to bond the VCSEL to the CMOS substrate. Electrical connections between the circuit and VCSEL were made with gold wire-bonds. The semiconductor parameter analyzer was used to run a voltage sweep on the 
(a)

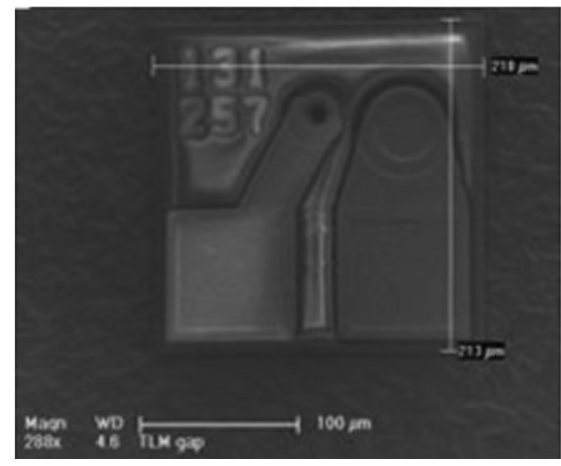

(b)

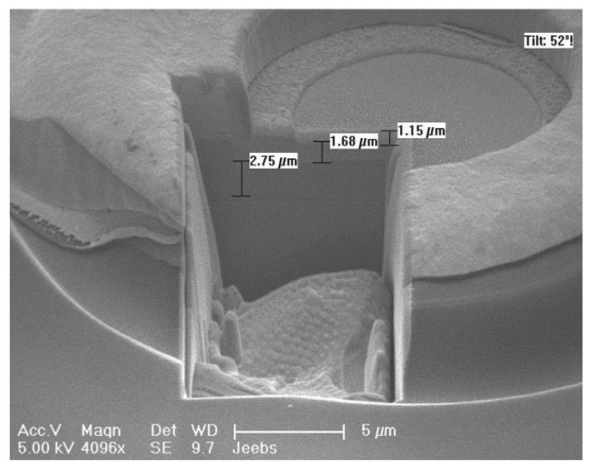

Fig. 11 (a) Vertical cavity surface emitting laser (VCSEL) chip after etching and (b) cross section of the active area of the device.
Table 1 Photovoltaic (PV) response of deep NWell-based complimentary metal oxide semiconductor devices.

\begin{tabular}{lcccc} 
Diode & $\begin{array}{c}\text { Open circuit } \\
\text { voltage }\end{array}$ & $\begin{array}{c}\text { Short circuit } \\
\text { current (D1) }\end{array}$ & $\begin{array}{c}\text { Short circuit } \\
\text { current (D2) }\end{array}$ & $\begin{array}{c}\text { Short circuit } \\
\text { current (D3) }\end{array}$ \\
\hline $\begin{array}{l}\text { Deep } \\
\text { NWell }\end{array}$ & $0.75 \mathrm{~V}$ & $50 \mu \mathrm{A}$ & $200 \mu \mathrm{A}$ & $500 \mu \mathrm{A}$ \\
Substrate & $0.6 \mathrm{~V}$ & $250 \mu \mathrm{A}$ & $1 \mathrm{~mA}$ & $1.5 \mathrm{~mA}$ \\
Surface & $0.7 \mathrm{~V}$ & $40 \mu \mathrm{A}$ & $150 \mu \mathrm{A}$ & $375 \mu \mathrm{A}$ \\
2 diodes & $1.1 \mathrm{~V}$ & $30 \mu \mathrm{A}$ & $110 \mu \mathrm{A}$ & $230 \mu \mathrm{A}$ \\
3 diodes & $1.4 \mathrm{~V}$ & $25 \mu \mathrm{A}$ & $75 \mu \mathrm{A}$ & $120 \mu \mathrm{A}$ \\
\hline
\end{tabular}

Fig. 12 PV response of a single surface diode.

VCSEL and measure its response. An external signal generator was used to generate a bit stream to be transmitted by the VCSEL. A Thorlabs PIN photodetector (FPD510) was used to measure the resulting signal which was fed to an oscilloscope for display.

The results from optical testing of the PV modules are presented in Fig. 12 for an individual surface diode. The device was illuminated with the $805-\mathrm{nm}$ IR laser and the response was measured using the source meter.

The response for all the devices in the deep NWell-based design was measured under the same conditions and is summarized in Table 1 . Here, D1 denotes a $30 \mu \mathrm{m} \times 30 \mu \mathrm{m}$ surface diode, D2 denotes a $100 \mu \mathrm{m} \times 100 \mu \mathrm{m}$ surface diode, and D3 denotes a $500 \mu \mathrm{m} \times 500 \mu \mathrm{m}$ surface diode. Electrical stacking of a surface diode and a deep NWell diode is referred to as " 2 diodes" and the stack of all three devices, i.e., surface, deep NWell, and substrate device is referred to as "3 diodes."

Different tissue thicknesses were tested and compared against each other to understand the effect of implantation depth on system performance. Typical results for a 3-mm thick chicken skin and tissue are plotted, along with results in air, in Fig. 13. It is expected that the subtracting effects due to the tissue can be minimized by using a better focusing mechanism (e.g., an integrated lens) or by an improved in vivo focusing mechanism. ${ }^{37}$

These results show the output efficiency to be around $0.4 \%$ which is reasonably close to the calculated value of $0.27 \%$. The

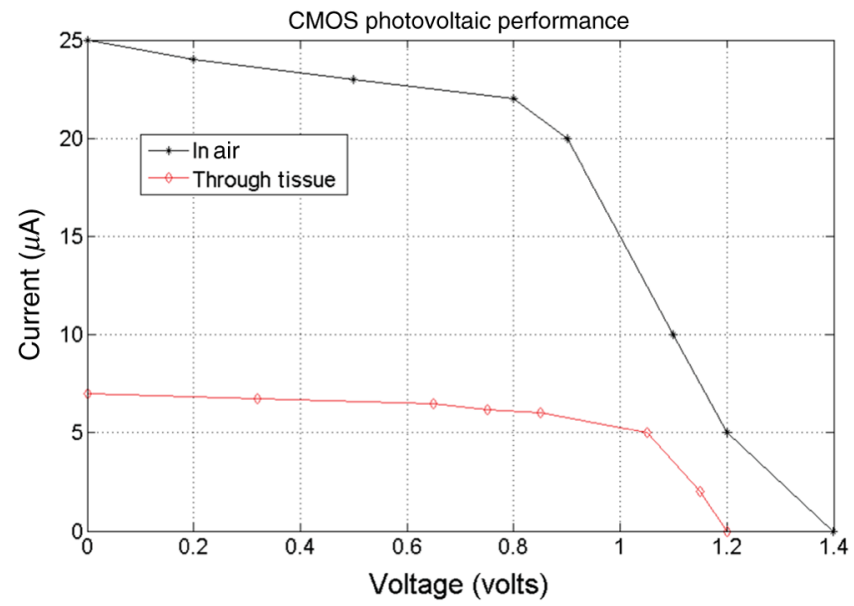

Fig. 13 PV response of a stacked CMOS NWell device.

difference can most likely be attributed to the variance between the theoretical and actual parameters for the skin sample used in the study.

The received power can be increased by improving the focus of the light source using a focusing lens, as shown by results in Table 2. It was observed that further delivered power enhancement was possible, but the photon intensity was high enough to cause tissue damage. 
Table 2 Effect of laser focusing on performance of deep NWell PV system.

\begin{tabular}{lcc} 
Diode & Open circuit voltage & Short circuit current (D1) \\
\hline Deep NWell & $0.8 \mathrm{~V}$ & $70 \mu \mathrm{A}$ \\
Substrate & $0.6 \mathrm{~V}$ & $350 \mu \mathrm{A}$ \\
Surface & $0.7 \mathrm{~V}$ & $65 \mu \mathrm{A}$ \\
2 Diodes & $1.1 \mathrm{~V}$ & $42 \mu \mathrm{A}$ \\
3 Diodes & $1.4 \mathrm{~V}$ & $30 \mu \mathrm{A}$ \\
\hline
\end{tabular}

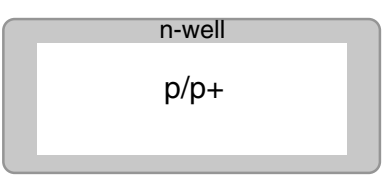

(a)

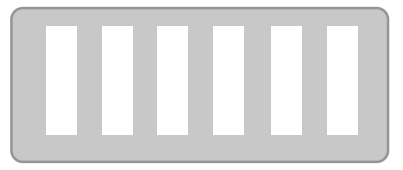

(c)

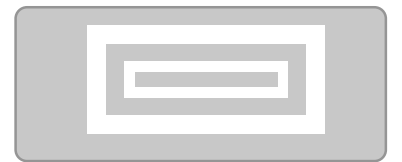

(b)

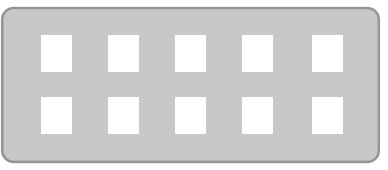

(d)
Fig. 14 Photodiode geometries for the CMOS PV design. The gray area denotes the $n$-doped well and the white area denotes $p / p+$ doped areas: (a) SD1, (b) SD2, (c) SD3, and (d) SD4.

Another measurement was done to compare the performance of the photodiodes with different geometries, as shown in Fig. 14. It was evident that the design consisting of distributed structures with higher peripheral areas around the $\mathrm{p}-\mathrm{n}$ junction provide higher output current. Further optimization using rigorous mathematical modeling of the CMOS process parameters could be done to further enhance the PV system efficiency.

The results from the geometry comparison are listed in Table 3. As discussed before, the best geometry (SD4 in Fig. 14) with the same geometrical area is $30 \%$ more efficient compared to the standard geometry (SD1) which is normally used for $\mathrm{p} / \mathrm{n}$ diodes in the CMOS device library.

For optical telemetry, the 850-nm wavelength VCSEL was tested before and after etching of its bulk GaAs substrate. A typical output from the VCSEL is shown in Fig. 15. As can be seen in the $I-V$ curves, the threshold voltage remained the same after etching away the substrate, and for the same driving

Table 3 Photodiode geometry comparison.

\begin{tabular}{lcc} 
Diode & Open circuit voltage & Short circuit current \\
\hline Surface diode 1 (SD1) & $0.7 \mathrm{~V}$ & $30 \mu \mathrm{A}$ \\
Surface diode 2 (SD2) & $0.66 \mathrm{~V}$ & $41 \mu \mathrm{A}$ \\
Surface diode 3 (SD3) & $0.63 \mathrm{~V}$ & $44 \mu \mathrm{A}$ \\
Surface diode 4 (SD4) & $0.6 \mathrm{~V}$ & $50 \mu \mathrm{A}$ \\
\hline
\end{tabular}

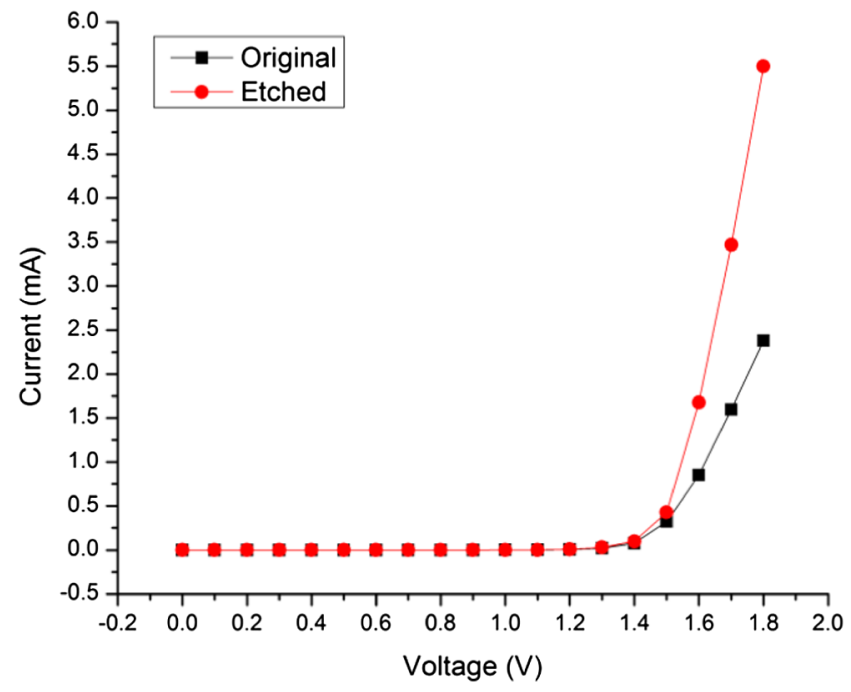

Fig. $15 I-V$ curves of a VCSEL chip before and after etching.

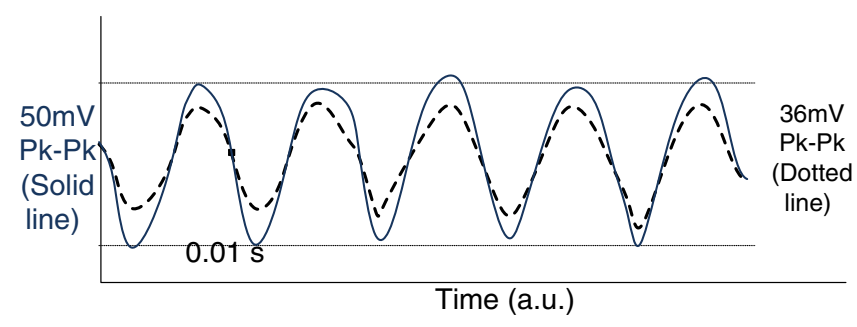

Fig. 16 VCSEL output before (dotted line) and after (solid line) power laser illumination.

voltage past the threshold, the current increased for the etched VCSEL, possibly due to less leakage into the substrate.

As noted earlier, a high-power laser $(0.8 \mathrm{~W}, 805 \mathrm{~nm})$ was used to power the integrated PV power supply. Due to scattering in the skin and tissue, some of this high-power laser may also illuminate the VCSEL. Experiments were performed to find the effects of such high intensities on the low-power VCSEL telemetry link. It was found that the power input laser increased the amplitude of the VCSEL signal (approximately 20\% to 30\%) and showed no negative effect on the communication signal from the VCSEL, as shown in Fig. 16.

We speculate that the increase in signal amplitude is due to the optical pumping of the VCSEL by the laser. This does not increase the signal level to a very large extent because the front mirror in the VCSEL design reduces the intensity that reaches the active medium. This shows that the optical components of the system (power and communication) can be used simultaneously as the power signal does not adversely affect the telemetry signal.

\section{Conclusion}

In this work, we demonstrated the use of optical links for both power transfer and communication for fully wireless implants. We provided the details of a system design which demonstrates that the "therapeutic window" in the NIR range can be effectively used for power transfer and data telemetry for such implants. Since this wavelength window falls within the range of the silicon absorption band, simple CMOS-based devices can 
be used for miniaturization of the overall system. This has the added advantage that silicon-based CMOS electronics can be used for extreme miniaturization of the overall device. Furthermore, since silicon is not an efficient material to modulate optical signals, we added compound semiconductor-based microlasers for telemetry transfer as their wavelength range also falls within the "therapeutic window." Next, we showed that sufficiently high voltages and currents can be generated from miniaturized CMOS integrated PV devices if concentrated laser light is used as a power source and high-quality junction diodes (large n, small reverse saturation current) are used as PV devices. Photogenerated voltage can further be increased if deep NWell designs are utilized. In this regard, we presented a topology which enables connecting three diodes in series in deep NWell-based CMOS processes to achieve high voltages. We also showed that high currents can be generated using substrate diodes owing to the large absorption region in these devices while still maintaining an overall small lateral footprint. We also demonstrated that these optical schemes can be used to transfer enough power, e.g., subcutaneous implant and that optical telemetry can be achieved using an ultrasmall VCSEL bonded to the CMOS platform. We also showed that such communication is possible in the presence of a large background illumination from a high-power laser as long as the power and communication wavelengths are sufficiently far away from each other, while still being within the "therapeutic window." This makes it possible to use completely optical methods for fully wireless implants and hence advances the techniques available for extreme miniaturization of implants which can eventually lead to long-term applications of such devices in many clinical applications.

\section{References}

1. I. L. Jones et al., "The potential of microelectrode arrays and microelectronics for biomedical research and diagnostics," Anal. Bioanal.Chem. 399, 2313-2329 (2011).

2. C. Ming Li et al., Implantable Electrochemical Sensors for Biomedical and Clinical Applications: Progress, Problems, and Future Possibilities, Vol. 14, Bentham Science Publishers, Emirate of Sharjah, United Arab Emirates (2007).

3. W. K. Ward et al., "The effect of microgeometry, implant thickness and polyurethane chemistry on the foreign body response to subcutaneous implants," Biomaterials, 4185-4192 (2002).

4. A. Yakovlev, S. Kim, and A. Poon, "Implantable biomedical devices: wireless powering and communication," IEEE Commun. Mag. 50(4), 152-159 (2012)

5. L. Chang et al., "Small-volume solution current-time behavior study for application in reverse iontophoresis-based non-invasive blood glucose monitoring," Sci. China Chem. 54, 223-230 (2011).

6. L. Chang et al., "Magnetic tweezers-based 3D microchannel electroporation for high-throughput gene transfection in living cells," Small 11, 1818-1828 (2015).

7. W. J. Heetderks, "RF powering of millimeter- and submillimeter-sized neural prosthetic implants," IEEE Trans. Biomed. Eng. 35, 323-327 (1988).

8. L. Yang et al., "RF powering for embedded glaucoma sensors in miniature packages," in Proc. Union Radio Scientifique Internationale General Assembly, Chicago, Illinois (2008).

9. M. H. Nazari, M. Mujeeb-U-Rahman, and A. Scherer, "An implantable continuous glucose monitoring microsystem in $0.18 \mu \mathrm{m}$ CMOS," in Presented at the VLSI Symp., Hawaii (2014).

10. S. Kim et al., "Wireless power transfer to a cardiac implant," Appl. Phys. Lett. 101, 1-4 (2012).

11. S. M. Sze and K. K. Ng, Physics of Semicondcutor Devices, John Wiley \& Sons, Hoboken, New Jersey (2007).
12. S. Ayazian and A. Hassibi, "Delivering optical power to subcutaneous implanted devices," in 33rd Annual Int. Conf. of the IEEE EMBS (2011).

13. D. M. Ackermann, Jr. et al., "Designing the optical interface of a transcutaneous optical telemetry link," IEEE Trans. Biomed. Eng. 55, 1365-1373 (2008).

14. M. Mujeeb-U-Rahman et al., "Nanofabrication techniques for fully integrated sensing platforms," in Nanotech, pp. 73-76 (2013).

15. B. Razavi, CMOS Analog Integrated Circuits, Mcgraw-Hill, New York (2001).

16. M. Sawan and B. Gosselin, "CMOS circuits for biomedical implantable devices," in VLSI Circuits for Biomedical Applications, K. Iniewski, Ed., Artech House, Norwood, Massachusetts (2008).

17. F. Yildiz, "Potential ambient energy-harvesting sources and techniques," J. Technol. Stud. 35, 40-48 (2009).

18. E. D. Palik, Handbook of Optical Constants of Solids, Goodwin House Publishers, New York and Boston (1997).

19. J. Jasper, "Layers of human skin," http://sketchup.google.com/3dware house/details?mid=fb67c58b41a468dc2347fdb8269abb0\&prevstart=0 (15 February 2014).

20. C. R. Simpson et al., "Near-infrared optical properties of ex vivo human skin and subcutaneous tissues measured using the Monte Carlo inversion technique," Phys. Med. Biol. 43, 2465-2478 (1998).

21. P. Rossetti et al., "Estimating plasma glucose from interstitial glucose: the issue of calibration algorithms in commercial continuous glucose monitoring devices," Sensors 10, 10936-10952 (2010).

22. A. N. Bashkatov et al., "Optical properties of human skin, subcutaneous and mucous tissues in the wavelength range from 400 to $2000 \mathrm{~nm}$," J. Phys. Dermatol. Appl. Phys. 38, 2543-2555 (2005).

23. B. C. Larson, "An optical telemetry system for wireless transmission of biomedical signals across the skin," PhD Dissertation, Dept. Elect. Eng., Massachusetts Institute of Technology, Cambridge, Massachusetts (1999).

24. I. V. Meglinski and S. J. Matcher, "Quantitative assessment of skin layers absorption and skin reflectance spectra simulation in the visible and near-infrared spectral regions," Physiol. Meas. 23, 741-753 (2002).

25. C. Rebecca Simpson et al., "Near-infrared optical properties of ex vivo human skin and subcutaneous tissues measured using the Monte Carlo inversion technique," Phys. Med. Biol. 43, 2465-2478 (1998).

26. S. Ayazianmavi, Photovoltaic (PV) and Fully-Integrated Implantable CMOS ICs, University of Texas, Austin (2012).

27. A. Krishnaswamy and G. Baranoski, A Study on Skin Optics, University of Waterloo, Canada (2004).

28. G. Zonios, J. Bykowski, and N. Kollias, "Skin melanin, hemoglobin, and light scattering properties can be quantitatively assessed in vivo using diffuse reflectance spectroscopy," J. Investig. Dermatol. 117, 1452-1457 (2001).

29. M. Mujeeb-U-Rahman, C. F. Chang, and A. Scherer, "Optical methods for wireless implantable sensing platforms," Proc. SPIE 8812, 88120M (2013).

30. U-L-M Photonics, "5Gbps 850nm VCSEL chip," 2013, http://www. photonics.philips.com/ (March 2014).

31. Thorlabs, "FPD510 series of high sensitivity PIN photodetectors: DC $200 \mathrm{MHz}$," 2012, http://www.thorlabs.us/catalogpages/V21/1541.PDF (20 Febraury 2014).

32. K. Murari et al., "Which photodiode to use: a comparison of CMOScompatible structures," IEEE Sens. J. 9, 752-760 (2009).

33. M. K. Law et al., "High-voltage generation with stacked photodiodes in standard CMOS process," IEEE Electron. Device Lett. 31, 1425-1427 (2010).

34. O. Aktan et al., "Optoelectronic CMOS power supply unit for electrically isolated microscale applications," IEEE J. Sel. Top. Quantum Electron. 17, 747-756 (2011).

35. K. I. Inoue et al., "Transcutaneous optical telemetry system using laser diode," Jpn J. Artif. Organs 27, 363-367 (1998).

36. J. L. Jewell et al., "Vertical-cavity surface-emitting lasers-design, growth, fabrication, characterization," IEEE J. Quantum Electron. 27, 1332-1346 (1991).

37. I. M. Vellekoop and C. M. Aegerter, "Focusing light through living tissue," Proc. SPIE 7554, 755430 (2010).

Muhammad Mujeeb-U-Rahman received his MS and PhD degrees in electrical engineering at Caltech in 2010 and 2014, respectively. 
His research is focused on developing novel, fully integrated wireless sensing platform for complex biochemical environments. He received the Demetriades-Tsafka-Kokkalis Prize for best work in nanotechnology at Caltech in 2014. He also received a Fulbright fellowship in 2008 for his MS at Caltech.

Dvin Adalian received his BS degree in physics from the California Institute of Technology in 2009. His research was focused on fabrication of microfluidic and MEMS devices. Currently, he is a PhD student in applied physics at Caltech and works on microfabrication of chemical sensors and silicon nanostructures.

Chieh-Feng Chang joined the Caltech Nanofabrication Group with the International Fulbright Science and Technology award and received his $\mathrm{PhD}$ in electrical engineering under the supervision of Prof. Axel Scherer in 2015. His research interest is in wired and wireless sensors of various configurations that combine nanofabrication and biophotonics for molecular and biomedical detection applications.

Axel Scherer is the Neches professor of electrical engineering, applied physics, and medical engineering at Caltech. His research focuses on the design and microfabrication of different devices. He pioneered the development of the first monolithic vertical cavity lasers. He has cofounded several companies in the area of silicon photonics and biomedical diagnostics, and leads a productive group focused on the miniaturization and integration of fluidic, optical, electronic, and magnetic devices for applications in biotechnology. 